\title{
Emission spectrum of broadband quantum dot superluminescent diodes
}

\author{
F. Friedrich, ${ }^{1, *}$ W. Elsäßer, ${ }^{1}$ and R. Walser ${ }^{1}$ \\ ${ }^{1}$ Institut für Angewandte Physik, Technische Universität Darmstadt, 64289 Darmstadt, Germany
}

(Dated: May 16, 2019)

\begin{abstract}
We present a microscopic theory of the amplified spontaneous emission of a spectrally broadband quantum dot superluminescent diode within the quantum white noise limit. From this multimode quantum theory, we have the ability to obtain all orders of temporal correlation functions. In particular, we derive rate equations for the optical power densities, the level occupation of inhomogeneous ensemble of quantum dots within the diode, as well as the emitted optical spectra. As the main result, we find the external power spectrum as a convolution of the intra-diode photon spectrum with a Lorentzian response. Assuming a Gaussian light-matter coupling results in a similar shaped Gaussian output spectrum, which agrees very well with available experimental data.
\end{abstract}

Keywords: quantum dot superluminescent diodes, power spectrum, ASE, amplified spontaneous emission, photon statistics, quantum dot, quantum fluctuation, Ito formalism, input-output formalism, quantum stochastic differential equations, multimode laser theory, rate equations, correlation function.

\section{INTRODUCTION}

Commercial devices for optical coherence tomography [2 4] and telecommunication [1] greatly benefit from the appealing features of spectrally broadband light-emitting quantum dot superluminescent diodes (QDSLDs). In such a QDSLD, light is generated in the regime of amplified spontaneous emission (ASE), i. e. right at the border between spontaneous and stimulated emission. Careful design of waveguide geometry and gain medium results in very high output intensities, spatial coherence and a very broad radiation spectrum width in the $\mathrm{THz}$ regime [5, [6], thus combining spatial coherence features of typical laser diodes with the remarkable broadband spectrum of light-emitting diodes.

Modern applications like ghost imaging $7-10]$ or the analysis of fundamental quantum optical questions on the photon statistics 11 15 benefit from these semiconductor devices. In 2011, Blazek et al. [16] measured temporal second-order correlation function on the fs time scale with fast two-photon detectors [17]. They discovered a novel state of light, which features a reduction of the second-order degree of coherence $g^{(2)}(\tau=0)$ from 2 to 1.33 at a temperature of $190 \mathrm{~K}$ without any spectral narrowing (spectral width remains $\mathrm{THz}$ ). Thus, these novel states of light are simultaneously incoherent in firstand coherent in second-order correlation function. Understanding this 'hybrid coherent light' is an interesting topic and provides more insights into the quantum nature of light-emitting broadband semiconductor sources.

Originally, amplified spontaneous emission has been already studied by Allen and Peters [18 21] in 1971. Amongst others, they formulated the ASE threshold condition and investigated spatial coherence properties of a classical field propagating in a bulk medium in the absence of an external cavity. The global spreading of LED illumination technology has revived the interest in

* Corresponding author:franziska.friedrich@physik.tu-darmstadt.de the radiation properties of diodes, for example studies of the emission and photon statistical characteristics of QDSLDs 22, 23]. Numerical models have been developed based on rate equations [24, 25], traveling wave approaches [26] and finite element methods [27].

Microscopic theories describing ASE are mostly focused on the specific semiconductor properties [28, 29]. However, hybrid coherent light generation is an optical process and should not require detailed information about semiconductor charge carrier dynamics. Thus, a quantum optical analysis of ASE adapted to superluminescent diodes with tilted end facets using a quantum dot gain medium is appropriate.

In a previous study of the optical spectral properties of such QDSLDs, a multimode phase-randomized Gaussian state was postulated as a premise to match the observed spectral shape [30]. One finds that the second-order degree of coherence is inversely proportional to the number of available modes, i.e. $g^{(2)}(\tau)$ is affected by the characteristics of each individual QDSLD. This prediction was experimentally confirmed by an optical feedback experiment [30, 31]. Further evidence on the nature of the optical quantum state came from an experiment superimposing a coherent single-mode laser beam with the broadband radiation of the QDSLD. The observed spectra agree with the theoretical analysis very well.

In order to shed more light on the nature of the photonic state, we will discuss in this article a microscopic model of the QDSLD, based on the input-output formalism of laser theory [32, 33]. Based on this multimode theory, we determine temporal first-order correlation function of the radiation field measured by a single-photon detector and compare them with the experimental data.

The article is organized as follows: after introducing basic facts of QDSLDs in Sec. III, we establish a theoretical model, where we consider the energy of the intrawaveguide system and set up the Ito quantum stochastic differential equations for the diode system. Within the rate equation limit, we study in Sec. III the stationary photon number as a function of the incoherent pumping 
rate for the special cases: 1) a single ASE field mode and 2) a multimode radiation field, both coupled to a homogeneous ensemble of quantum dots. In Sec. IV] we use the input-output formalism to analyze the first-order autocorrelation function directly related to the output spectral density of light-emitting QDSLDs. A conclusion is given in Sec. V Three appendices contain details on the quantum Ito calculus in App. A the multi-channel transmission spectrum of a passive waveguide in App. B as well as the interpolation procedure of the power spectrum in App. C.

\section{PROPERTIES OF QUANTUM DOT SUPERLUMINESCENT DIODES}

Superluminescent diodes 34, 35] are optoelectronic semiconductor devices emitting spatially directed light with spectral widths in the $\mathrm{THz}$ regime. A typical measured optical power spectrum [36] of a QDSLD is shown in Fig. 1] where experimental data are compared with a Gaussian fit (Eq. (C1)).

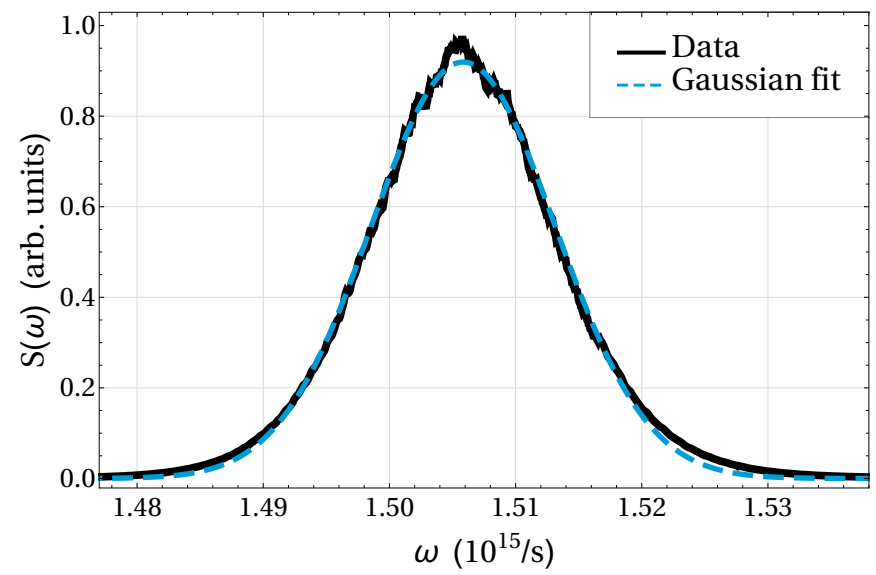

Figure 1: Typical shape of an experimental QDSLD power spectrum $S(\omega)$ (black, solid line) versus angular frequency $\omega$. Central frequency $\bar{\omega}=2 \pi \cdot 0.24 \mathrm{PHz}$ $(\bar{\lambda}=1249.14 \mathrm{~nm})$, standard deviation $\sigma=2 \pi \cdot 1.16 \mathrm{THz}$ and spectral width $b=2 \pi \cdot 4.11 \mathrm{THz}(\Delta \lambda=21.39 \mathrm{~nm})$ are obtained from a Gaussian fit (blue, dashed line, Eq. (C1).

In the setup of Ref. [16], a tilted optical waveguide was used (angle $5-8^{\circ}$ with respect to the longitudinal axes). The emission facets where anti-reflection coated to suppress the formation of longitudinal modes [37]. The gain material of a QDSLD consists of quantum dots differing in size, shape and material composition, leading to a considerable inhomogeneous level-broadening. In order to obtain high efficient light amplifications with broad spectral ranges 5 to 10 layers of the gain medium are essential, where each layer consists of about 400 quantum $\operatorname{dots} / \mu^{2}$. They are spatially separated by other semiconductor materials, the so-called buffer layers having a refractive index, $n_{m}=3.5$, slightly smaller than the index of the single gain layers, $n_{c}=3.505$ [38].

\section{MODEL OF A QUANTUM DOT SUPERLUMINESCENT DIODE}

Let us consider a simple diode model with one layer of gain medium, as depicted in Fig. 2. There, the gain medium consists of $M$ quantum dots, which are embedded in a bulk material that defines a waveguide.

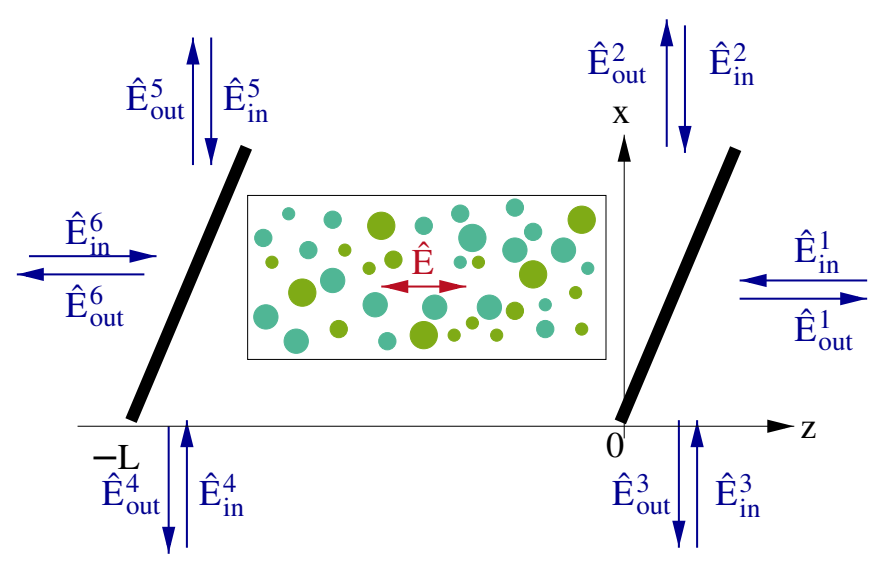

Figure 2: Model of a QDSLD (top view): bulk material in a rectangular waveguide hosts $M$ quantum dots. The tilted end facets of the QDSLD prevent reflection back into the medium and are formally represented by beam splitters. In principle, six external quantum channels $\hat{\mathbf{E}}^{\alpha}$ with $\alpha=1, \ldots, 6$ couple into the waveguide and interact with the ASE field $\hat{\mathbf{E}}$.

\section{A. ASE field}

We now study the broadband light inside the diode which must be described by a multimode electrical field, $\hat{\mathbf{E}}=\hat{\mathbf{E}}^{(+)}+\hat{\mathbf{E}}^{(-)}$with positive frequency part

$$
\hat{\mathbf{E}}^{(+)}(\mathbf{r}, t)=\sum_{\left\{k_{i}\right\}} \mathbf{u}_{i}(\mathbf{r}) \hat{a}_{i}(t) .
$$

As we want to pursue a quantum theory of light, we need to consider a Hamilton operator

$$
\hat{\mathcal{H}}_{r}=\sum_{\left\{k_{i}\right\}} \hbar \omega_{i} \hat{a}_{i}^{\dagger} \hat{a}_{i},
$$

depending on the bosonic amplitudes $\hat{a}_{i}$, which satisfy the commutation relation $\left[\hat{a}_{i}, \hat{a}_{j}^{\dagger}\right]=\delta_{i j}$. The electric field in Eq. (1) is formed by a superposition of $N$ modes of type $\mathbf{u}_{i}(\mathbf{r})=\mathcal{E}_{i} \chi(x, y) e^{\mathrm{i} k_{i} z} \mathbf{e}_{y}$, which are solutions of the Helmholtz equation

$$
\left(\Delta+k_{i}^{2}\right) \mathbf{u}_{i}(\mathbf{r})=0, \quad \omega_{i}=\left|k_{i}\right| \frac{c}{n_{c}}
$$


with rectangular geometry. They factorize into a single transverse wave function $\chi(x, y)$ and longitudinal plane waves with wave numbers $\left|k_{i}\right|$ with $k_{i}=2 \pi i n_{c} / L$. The length of the waveguide is denoted by $L$. Due to the rectangular geometry of the QDSLD [39], the field is linearly polarized in $y$-direction. The transverse mode function is normalized to the cross-section area $A=\int_{A} d^{2} x|\chi|^{2}$. If the system volume is $V=A L$, then the normalization factor of the electric field reads $\mathcal{E}_{i}=\mathrm{i} \sqrt{\hbar \omega_{i} / 2 \epsilon V}$. According to Eq. (3), there is a linear dispersion relation between frequency and wave number with vacuum speed of light $c$.

\section{B. External fields}

The input-output arrangement of the diode system depicted in Fig. 2 couples the internal ASE field $\hat{\mathbf{E}}$ to the outside. There, six outside channels $\alpha \in\{1, \ldots, 6\}$

$$
\hat{\mathbf{E}}^{\alpha(+)}(\mathbf{r}, t)=\sum_{\left\{k_{i}\right\}} \sum_{q \in M_{i}^{\alpha}} \mathbf{v}_{q}^{\alpha}(\mathbf{r}) \hat{b}_{i q}^{\alpha}(t),
$$

are themselves multimode fields with amplitudes $\hat{b}_{i q}^{\alpha}$ oscillating at frequency $\omega_{q}=c\left|k_{q}\right|$ of frequency group $M_{i}^{\alpha}$. The corresponding Hamilton operator is given by

$$
\hat{\mathcal{H}}_{c}=\sum_{\alpha=1}^{6} \sum_{\left\{k_{i}\right\}} \sum_{q \in M_{i}^{\alpha}} \hbar \omega_{q} \hat{b}_{i q}^{\alpha \dagger} \hat{b}_{i q}^{\alpha} .
$$

For simplicity, we neglect spatial expansion of the electromagnetic field emitted by the diode and consider only a one-dimensional propagation. Clearly, from an experimental point of view this can be achieved by single-mode fibers, which support linearly polarized, single modes

$$
\begin{aligned}
\mathbf{v}_{q}^{\alpha}(\mathbf{r}) & =\mathcal{E}_{q} \mathbf{e}_{y}\left\{\begin{array}{ll}
\xi(x, y) e^{\mathrm{i} k_{q} z} & \alpha=1,6 \\
\xi(y, z) e^{\mathrm{i} k_{q} x} & \alpha=2,3,4,5
\end{array},\right. \\
\mathcal{E}_{q} & =\mathrm{i} \sqrt{\frac{\hbar \omega_{q}}{2 \epsilon_{0} V}}
\end{aligned}
$$

as well as a transverse mode function $\xi$ that is normalized to the cross-section area $A=\int_{A} d^{2} x|\xi|^{2}$. We define frequency groups of outside modes

$$
M_{i}^{\alpha}=\left\{q \in \mathbb{Z}: q_{\alpha}^{\min }\left(k_{i}\right) \leq q \leq q_{\alpha}^{\max }\left(k_{i}\right)\right\},
$$

with equidistant frequency spacing $\Delta \omega=2 \pi \Delta \nu$ which couple to individual ASE field modes at frequency $\omega_{i}$ in the sense of well resolved bands. To be specific, we specify the set of modes which couple an ASE mode $i$ to $\hat{b}_{i q}^{\alpha}(t), q \in M_{i}^{\alpha}$, which satisfy the bosonic commutation relation

$$
\left[\hat{b}_{i q}^{\alpha}(t), \hat{b}_{j p}^{\beta \dagger}(t)\right]=\delta_{\alpha \beta} \delta_{q p} \delta_{i j} .
$$

Here, correlations between the channel fields are excluded within our theoretical considerations.
Assuming that single frequencies $\omega_{q}$ are centered around a certain frequency $\omega_{i}$, that is $\omega_{q} \approx \omega_{i}$, so that $\mathcal{E}_{q} \approx \mathcal{E}_{i}$ (see Eq. (77) and introducing the free input- and output fields,

$$
\begin{aligned}
\hat{b}_{i, \text { in }}^{\alpha}(t) & \equiv \sqrt{\Delta \nu} \sum_{q \in M_{i}^{\alpha}} e^{-\mathrm{i} \omega_{q}\left(t-t_{i}\right)} \hat{b}_{i q}^{\alpha}\left(t_{i}\right), \\
\hat{b}_{i, \text { out }}^{\alpha}(t) & \equiv \sqrt{\Delta \nu} \sum_{q \in M_{i}^{\alpha}} e^{-\mathrm{i} \omega_{q}\left(t-t_{f}\right)} \hat{b}_{i q}^{\alpha}\left(t_{f}\right),
\end{aligned}
$$

in terms of their initial and final values at $t_{i}$ and $t_{f}$, respectively, the positive frequency part of the electric field from channel 1 emitted on the right end facet $(z>0)$ reads 33

$$
\begin{gathered}
\hat{\mathbf{E}}^{1(+)}(\mathbf{r}, t)=\hat{\mathbf{E}}_{\text {in }}^{1(+)}\left(x, y, \tau^{-}\right)+\hat{\mathbf{E}}_{\text {out }}^{1(+)}\left(x, y, \tau^{+}\right), \\
\hat{\mathbf{E}}_{\text {in } / \text { out }}^{(+)}(x, y, \tau)=\sum_{\left\{k_{i}>0\right\}} \mathbf{v}_{i}^{1}(x, y) \hat{b}_{i, \text { in } / \text { out }}^{1}(\tau),
\end{gathered}
$$

with $\tau^{ \pm}=t \pm z / c$ and $\mathbf{v}_{i}^{1}(x, y)=\mathcal{E}_{i} \xi(x, y) \mathbf{e}_{y} / \sqrt{\Delta \nu}$. The other optical channels $\hat{\mathbf{E}}^{\alpha}$ of Fig. 2 can be described in an analogous fashion, but are not required for this discussion.

Evaluating the frequency sum in the continuum limit (c.f. App. B), requires the frequency spacing $\Delta \nu$ as an integration measure. This procedure defines the "quantum white noise" limit [40],

$$
\left[\hat{b}_{i, \text { in } / \text { out }}^{\alpha}(t), \hat{b}_{j, \text { in } / \text { out }}^{\beta \dagger}\left(t^{\prime}\right)\right]=\delta_{i j} \delta_{\alpha \beta} \delta\left(t-t^{\prime}\right),
$$

for the input and output fields and demonstrates their infinitely short correlation time.

\section{Gain medium}

The gain medium is formed by an ensemble of $M$ quantum dots (QDs) embedded in semiconductor material. In order to achieve light amplification, we need to consider at least three levels [41]. The Hamiltonian of the QD system reads

$$
\hat{\mathcal{H}}_{a}=\sum_{j=1}^{M} \sum_{r=0}^{2} \hbar \omega_{r}^{j} \hat{\sigma}_{r r}^{j \dagger} \hat{\sigma}_{r r}^{j} .
$$

$\hat{\sigma}_{r r}^{j}$ denotes the population of level $|r\rangle$ of the $j$ th quantum dot. In Fig. 3, we show a closed three-level system with eigen-energies $\hbar \omega_{i}$ and transition frequencies $\omega_{i j}=\omega_{i}-$ $\omega_{j}$ between level $i$ and $j$.

Each quantum dot is incoherently pumped with rate $R$ and releases the energy via amplified spontaneous emission on the $|1\rangle \rightarrow|2\rangle$ transition to the multimode ASE field (11). We couple each quantum dot $j$ to a single phonon bath of energy

$$
\hat{\mathcal{H}}_{\mathrm{b}}^{j}=\sum_{\{s\}} \sum_{\{k\}} \hbar \omega_{s k}^{j} \hat{b}_{s k}^{j \dagger} \hat{b}_{s k}^{j}, \quad s \in\{(21),(20),(10)\},
$$




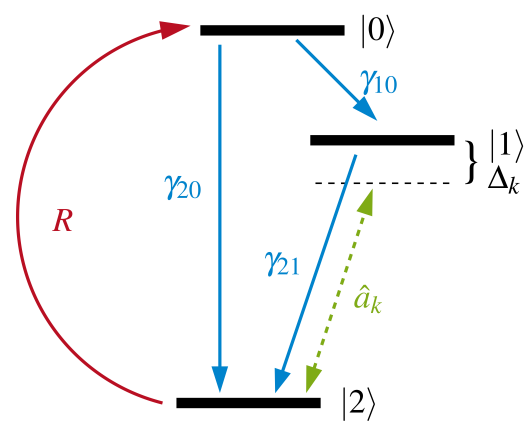

Figure 3: Three-level quantum dot energy diagram with decay rates $\gamma_{i j}(i, j \in\{0,1,2\})$, driven by the ASE radiation field with amplitudes $\hat{a}_{i}$ and incoherently pumped at rate $R$.

given by the sum of three independent baths, each acting on one particular quantum dot transition $s$. They satisfy the bosonic commutation relation

$$
\left[\hat{b}_{s k}^{j}(t), \hat{b}_{s^{\prime} k^{\prime}}^{j \dagger}(t)\right]=\delta_{k k^{\prime}} \delta_{s s^{\prime}} .
$$

Finally, we disregard interactions between quantum dots.

\section{Light-amplification by inhomogeneous broadened quantum dots}

The energy of the isolated diode system,

$$
\hat{\mathcal{H}}_{s}=\hat{\mathcal{H}}_{r}+\hat{\mathcal{H}}_{a}+\hat{\mathcal{H}}_{\text {int }},
$$

is given by the sum of the energy of the ASE field modes (21), the energy of the $M$ atomic system (15) and the dipole interaction between the broadband light field and the $M$ distinguishable quantum dots in the rotating wave approximation,

$$
\hat{\mathcal{H}}_{\text {int }}=-\mathrm{i} \sum_{\left\{k_{i}\right\}} \sum_{j=1}^{M} \hbar g_{i}^{j} \hat{\sigma}_{21}^{j \dagger} \hat{a}_{i}+\text { h.c. }
$$

with transition operator $\hat{\sigma}_{21}^{j}$ between level $|2\rangle$ and $|1\rangle$. The coupling constant, $g_{i}^{j}=-\mathbf{i} \mathbf{d}_{21}^{j *} \mathbf{u}_{i}\left(\mathbf{r}_{j}\right) / \hbar$, depends on frequency $\omega_{i}$ and is proportional to the dipole matrix element $\mathbf{d}_{21}^{j}$.

Switching to a suitable interaction picture

$$
\hat{H}_{s}=\mathrm{i} \hbar \dot{\hat{U}}^{\dagger} \hat{U}+\hat{U}^{\dagger} \hat{\mathcal{H}}_{s} \hat{U}
$$

with transformation operator

$$
\hat{U}=\exp (-\mathrm{i} \hat{G} t)
$$

where

$$
\hat{G}=\sum_{\left\{k_{i}\right\}} \bar{\omega}_{12} \hat{a}_{i}^{\dagger} \hat{a}_{i}+\sum_{j=1}^{M}\left[\omega_{0}^{j} \hat{\sigma}_{00}^{j}-\left(\omega_{1}^{j}-\delta \omega_{12}^{j}\right) \hat{\sigma}_{11}^{j}+\omega_{2}^{j} \hat{\sigma}_{22}^{j}\right],
$$

the time dependent Hamiltonian reads

$$
\begin{aligned}
\hat{H}_{s}= & \hbar \sum_{\left\{k_{i}\right\}} \Delta_{i} \hat{a}_{i}^{\dagger} \hat{a}_{i}+\hbar \sum_{j=1}^{M} \delta \omega_{12}^{j} \hat{\sigma}_{11}^{j} \\
& -\mathrm{i} \hbar \sum_{\left\{k_{i}\right\}} \sum_{j=1}^{M}\left(g_{i}^{j} \hat{\sigma}_{21}^{j \dagger} \hat{a}_{i}+\text { h.c. }\right) .
\end{aligned}
$$

In this interaction picture, the ASE field oscillates with detuning $\Delta_{i}=\omega_{i}-\bar{\omega}_{12}$, where $\bar{\omega}_{12}=\sum_{\left\{k_{i}\right\}} \omega_{i} / N$ is the mean transition frequency of the quantum dots. Accordingly, the frequency $\delta \omega_{12}^{j}=\omega_{12}^{j}-\bar{\omega}_{12}$ represents a small deviation of the $j$ th quantum dot from the mean value.

\section{E. Intrawaveguide field}

In order to describe the total QDSLD intrawaveguide system, we follow the concepts of C. Gardiner in 1985 [40, 42] dealing with open quantum systems. Using the Ito form of a quantum stochastic differential equation (QSDE) defined in (A1), the differential of the $i$ th ASE field mode in terms of the input noise operators reads

$$
\begin{aligned}
d \hat{a}_{i}= & -\mathrm{i}\left[\Delta_{i}-\mathrm{i} \gamma_{i}^{l r}\right] \hat{a}_{i} d t+\sum_{j=1}^{M} g_{i}^{j *} \hat{\sigma}_{21}^{j} d t \\
& -d \hat{B}_{i}^{l}-d \hat{B}_{i}^{r}, \quad \gamma_{i}^{l r}=\frac{1}{2}\left(\gamma_{i}^{l}+\gamma_{i}^{r}\right) .
\end{aligned}
$$

The first term in Eq. (24) describes the free evolution of the mode $i$ and includes the external damping rates $\gamma_{i}^{l}, \gamma_{i}^{r}$ resulting from the left and right input noise fields leading to damping effects of the system. The second term describes the interaction of the ASE field mode with all $M$ quantum dots and the last two terms consider noise fluctuations. According to Fig. 2, the left and right noise operators are given by

$$
d \hat{B}_{i}^{r}(t)=\frac{\zeta_{i}^{r *}}{2}\left(d \hat{b}_{i}^{1}+d \hat{b}_{i}^{2}\right), d \hat{B}_{i}^{l}(t)=\frac{\zeta_{i}^{l *}}{2}\left(d \hat{b}_{i}^{4}+d \hat{b}_{i}^{6}\right),
$$

with

$$
\zeta_{i}^{\alpha}=\sqrt{\gamma_{i}^{\alpha}} e^{\mathrm{i} \phi_{i}^{\alpha}}
$$

and normalized Ito increments $d b_{i}^{\alpha},(\alpha=1, \ldots, 6)$ satisfying the commutation relation,

$$
\left[d \hat{b}_{i}^{\alpha}(t), d \hat{b}_{j}^{\beta \dagger}\left(t^{\prime}\right)\right]=\delta_{i j} \delta_{\alpha \beta} \delta\left(t-t^{\prime}\right) d t d t^{\prime},
$$

as well as the Ito algebra listed in App. A. The damping rates on the left and right side of the beam splitter are defined by

$$
\gamma_{i}^{r} \equiv 2 \gamma_{i}^{1}=2 \gamma_{i}^{2}, \quad \gamma_{i}^{l} \equiv 2 \gamma_{i}^{4}=2 \gamma_{i}^{6} .
$$

Please note, the input fields of channel 3 and $5, \hat{\mathbf{E}}_{\text {in }}^{3}$ and $\hat{\mathbf{E}}_{\text {in }}^{5}$, never enter the waveguide. Therefore, the corresponding damping rates, proportional to the coupling 
strength between reservoir and ASE field, are equal to zero. Furthermore, we claim that all phases $\phi_{i}^{\alpha}$ of the channels from the left and right hand side are equal, i.e.

$$
\phi_{i}^{r} \equiv \phi_{i}^{1}=\phi_{i}^{2}, \quad \phi_{i}^{l} \equiv \phi_{i}^{4}=\phi_{i}^{6} .
$$

In the same way, we can derive the QSDE for the atomic coherences and populations of the quantum dots. Introducing a global incoherent pumping rate $R=n_{20}^{j} \gamma_{20}^{j}$ [43 45] acting equally strong on each individual quantum dot (see Fig. 3), the quantum dot populations and coherences in terms of the Ito increments $d \hat{b}_{m}^{j}=d \hat{B}_{m}^{j} / \sqrt{\gamma_{m}^{j}}$ read:

Populations:

$$
\begin{aligned}
d \hat{\sigma}_{00}^{j}= & {\left[-\Gamma_{0}^{j} \hat{\sigma}_{00}^{j}+\gamma_{10}^{j} n_{10}^{j} \hat{\sigma}_{11}^{j}+R \hat{\sigma}_{22}^{j}\right] d t } \\
& -\left(\hat{\sigma}_{10}^{j \dagger} d \hat{B}_{10}^{j}+\hat{\sigma}_{20}^{j \dagger} d \hat{B}_{20}^{j}+\text { h.c. }\right) \\
d \hat{\sigma}_{11}^{j}= & -\Gamma_{1}^{j} \hat{\sigma}_{11}^{j} d t-\sum_{\left\{k_{i}\right\}}\left(g_{i}^{j *} \hat{a}_{i}^{\dagger} \hat{\sigma}_{21}^{j} d t+\text { h.c. }\right) \\
& +\gamma_{10}^{j}\left(n_{10}^{j}+1\right) \hat{\sigma}_{00}^{j} d t+\gamma_{21}^{j} n_{21}^{j} \hat{\sigma}_{22}^{j} d t \\
& -\left(\hat{\sigma}_{21}^{j \dagger} d \hat{B}_{21}^{j}-\hat{\sigma}_{10}^{j \dagger} d \hat{B}_{10}^{j}+\text { h.c. }\right) \\
d \hat{\sigma}_{22}^{j}=- & \Gamma_{2}^{j} \hat{\sigma}_{22}^{j} d t+\sum_{\left\{k_{i}\right\}}\left(g_{i}^{j *} \hat{a}_{i}^{\dagger} \hat{\sigma}_{21}^{j} d t+\text { h.c. }\right) \\
+ & \gamma_{21}^{j}\left(n_{21}^{j}+1\right) \hat{\sigma}_{11}^{j} d t+\left(R+\gamma_{20}^{j}\right) \hat{\sigma}_{00}^{j} d t \\
+ & \left(\hat{\sigma}_{21}^{j \dagger} d \hat{B}_{21}^{j}+\hat{\sigma}_{20}^{j \dagger} d \hat{B}_{20}^{j}+\text { h.c. }\right)
\end{aligned}
$$

and coherences:

$$
\begin{aligned}
d \hat{\sigma}_{21}^{j}= & -\left[\mathrm{i}\left(\delta \omega_{12}^{j}-\mathrm{i} \Gamma_{21}^{j}\right) \hat{\sigma}_{21}^{j}+\sum_{\left\{k_{i}\right\}} g_{i}^{j} \hat{w}^{j} \hat{a}_{i}\right] d t \\
& +\hat{w}^{j} d \hat{B}_{21}^{j}+d \hat{B}_{10}^{j \dagger} \hat{\sigma}_{20}^{j}+\hat{\sigma}_{10}^{j \dagger} d \hat{B}_{20}^{j} \\
d \hat{\sigma}_{20}^{j}= & {\left[-\Gamma_{20}^{j} \hat{\sigma}_{20}^{j}+\sum_{\left\{k_{i}\right\}} g_{i}^{j} \hat{\sigma}_{10}^{j} \hat{a}_{i}\right] d t } \\
& +\hat{\sigma}_{10}^{j} d \hat{B}_{21}^{j}-\left(\hat{\sigma}_{22}^{j}-\hat{\sigma}_{00}^{j}\right) d \hat{B}_{20}^{j}-\hat{\sigma}_{21}^{j} d \hat{B}_{10}^{j} \\
d \hat{\sigma}_{10}^{j}= & {\left[\mathrm{i}\left(\delta \omega_{12}^{j}-\mathrm{i} \Gamma_{10}^{j}\right) \hat{\sigma}_{10}^{j}-\sum_{\left\{k_{i}\right\}} g_{i}^{j *} \hat{a}_{i}^{\dagger} \hat{\sigma}_{20}^{j}\right] d t } \\
& -d \hat{B}_{21}^{j \dagger} \hat{\sigma}_{20}^{j}-\hat{\sigma}_{21}^{j \dagger} d \hat{B}_{20}^{j}-\left(\hat{\sigma}_{11}^{j}-\hat{\sigma}_{00}^{j}\right) d \hat{B}_{10}^{j}
\end{aligned}
$$

with inversion $\hat{w}^{j}=\hat{\sigma}_{11}^{j}-\hat{\sigma}_{22}^{j}$ and decay rates

$$
\begin{aligned}
\Gamma_{0}^{j} & =\gamma_{10}^{j}\left(n_{10}^{j}+1\right)+\gamma_{20}^{j}+R, \\
\Gamma_{1}^{j} & =\gamma_{21}^{j}\left(n_{21}^{j}+1\right)+\gamma_{10}^{j} n_{10}^{j}, \\
\Gamma_{2}^{j} & =\gamma_{21}^{j} n_{21}^{j}+R, \\
\Gamma_{m n}^{j} & =\frac{1}{2}\left(\Gamma_{m m}^{j}+\Gamma_{n n}^{j}\right), m, n \in\{0,1,2\}, m \neq n .
\end{aligned}
$$

\section{F. On the nature of the quantum state of the QDSLD system}

Regarding the spectral measurements of highly incoherent broadband radiation fields (see Fig. 11), we assume a missing coherent amplitude of ASE mode $i$, that is

$$
\left\langle\hat{a}_{i}\right\rangle=\operatorname{Tr}\left\{\hat{a}_{i} \hat{\rho}\right\}=0 .
$$

Furthermore, we disregard correlations between different ASE field modes at the same space-time event, so that the average value,

$$
\left\langle\hat{a}_{i}^{\dagger} \hat{a}_{j}\right\rangle=n_{i} \delta_{i j}
$$

is given by the photon number $n_{i}$ of mode $i$. Regarding the input noise field, we do not allow interactions between the different external channel modes and between these modes with the quantum dot bath modes inside the waveguide. Occupation numbers and decay rates of the left and right hand side of the waveguide are assumed to be equal for all ASE field modes, that is $n_{i}^{\alpha}=n^{\alpha}$, $\gamma_{i}^{\alpha}=\gamma^{\alpha}$ with $\alpha=l, r$.

As the influence of a single quantum dot on the ASE radiation field mode $i$ is small, higher order corrections can be neglected by decorrelating field and atomic operator (decorrelation approximation) [46], i.e.

$$
\left\langle\hat{a}_{m}^{\dagger} \hat{w}^{j} \hat{a}_{n}\right\rangle \approx\left\langle\hat{a}_{m}^{\dagger} \hat{a}_{n}\right\rangle\left\langle\hat{w}^{j}\right\rangle .
$$

Furthermore, we adiabatically eliminate coherences as dynamical variables in the averaged QSDEs by replacing $\left\langle\hat{\sigma}_{21}^{j \dagger} \hat{a}_{i}\right\rangle$ by its stationary solution

$$
\left\langle\hat{\sigma}_{21}^{j \dagger} \hat{a}_{i}\right\rangle \approx \frac{g_{i}^{j *}\left(n_{i}\left\langle\hat{w}^{j}\right\rangle+\left\langle\hat{\sigma}_{11}^{j}\right\rangle\right)}{\mathrm{i} \Delta_{i}^{j}+\Gamma^{j}},
$$

with photon number $n_{i}$ of mode $i, \Gamma^{j} \equiv \Gamma_{21}^{j}+\gamma^{l r}$ with $\Gamma_{21}^{j}$ defined in Eq. (33) as well as a detuning $\Delta_{i}^{j}$ given by $\Delta_{i}^{j}=\Delta_{i}-\delta \omega_{12}^{j}=\omega_{i}-\omega_{12}^{j}$.

\section{G. Rate equations}

A measured optical power spectrum shows the behavior of the optical power as a function of angular frequency. The power itself is directly related to the stationary photon number which again is determined by the system's rate equations, whose deviation is easy to handle in the context of the Ito formalism. We only have to calculate the expectation value of the QSDE for the ASE field mode product $\hat{a}_{i}^{\dagger} \hat{a}_{j}$ and the populations of the $j$ th quantum dot, utilizing the characteristics of the Ito increments (see App. A). Applying the assumptions and approximations of section IIIF and regarding the fact that the Ito increments commute with the system operators at equal time, we obtain rate equations of the 
QDSLD

$$
\begin{aligned}
\dot{n}_{i}= & \sum_{j=1}^{M} \gamma G_{i}^{j}\left(n_{i} w^{j}+\sigma_{11}^{j}\right)-2 \gamma^{l r} n_{i}+n^{l} \gamma^{l}+n^{r} \gamma^{r} \\
\dot{\sigma}_{22}^{j}= & \sum_{\left\{k_{i}\right\}} \gamma G_{i}^{j}\left(n_{i} w^{j}+\sigma_{11}^{j}\right)-\Gamma_{22}^{j} \sigma_{22}^{j}+\left(R+\gamma_{20}^{j}\right) \sigma_{00}^{j} \\
& +\gamma_{21}^{j}\left(n_{21}^{j}+1\right) \sigma_{11}^{j} \\
\dot{\sigma}_{11}^{j}= & -\sum_{\left\{k_{i}\right\}} \gamma G_{i}^{j}\left(n_{i} w^{j}+\sigma_{11}^{j}\right)-\Gamma_{11}^{j} \sigma_{11}^{j}+\gamma_{21}^{j} n_{21}^{j} \sigma_{22}^{j} \\
& +\gamma_{10}^{j}\left(n_{10}^{j}+1\right) \sigma_{00}^{j} \\
\dot{\sigma}_{00}^{j}= & -\Gamma_{00}^{j} \sigma_{00}^{j}+\gamma_{10}^{j} n_{10}^{j} \sigma_{11}^{j}+R \sigma_{22}^{j},
\end{aligned}
$$

with mean population $\left\langle\hat{\sigma}_{i i}^{j}\right\rangle=\sigma_{i i}^{j}$ of level $|i\rangle$ of the $j$ th quantum dot and detuning $\Delta_{i}^{j}$. The "cooperativity" strength,

$$
G_{i}^{j} \equiv \frac{2}{\gamma} \frac{\left|g_{i}^{j}\right|^{2} \Gamma^{j}}{\Delta_{i}^{j 2}+\Gamma^{j 2}} \leq \frac{2\left|g_{i}^{j}\right|^{2}}{\gamma \Gamma^{j}},
$$

assesses the relative importance of the coherent atomic field coupling strength $g_{i}^{j}$ to the incoherent processes like pump rate $R$, external damping rates $\gamma^{l}, \gamma^{r}$ and atomic decay rates $\Gamma^{j}$. This coupled nonlinear equation system (44) can be solved numerically. However, in the following we have a closer look at two limiting cases: a single- and a multimode radiation field interacting with a homogeneous ensemble of quantum dots, where we assume negligible occupation numbers and damping rate, $n_{10}=0$, $n_{21}=0, \gamma_{20}=0$, vacuum input fields, $n^{r}=n^{l}=0$, and equal external damping rates $\gamma^{l}=\gamma^{r} \equiv \gamma$.

\section{Single-mode ASE field and identical quantum dots}

In case of a single-mode radiation field $(N=1)$ with frequency $\omega$ acting on transition $|1\rangle \leftrightarrow|2\rangle$ of $M$ equal quantum dots with transition frequency $\omega_{12}$, we find an analytical solution for the stationary photon number $n^{s}$,

$$
\begin{aligned}
n^{s} & =a\left(1+\sqrt{1+\frac{b}{a^{2}}}\right), \\
a & =\frac{\gamma_{10} R-\gamma_{21}\left(\gamma_{10}+R\right)-w_{c} \beta}{2 w_{c} \gamma G \alpha}, \quad b=\frac{\gamma_{10} R}{w_{c} \gamma G \alpha},
\end{aligned}
$$

with

$$
\begin{aligned}
& \alpha=3 R+2 \gamma_{10}, \\
& \beta=\left(\gamma \bar{G}+\gamma_{21}\right)\left(\gamma_{10}+2 R\right)+\gamma_{10} R
\end{aligned}
$$

and $\bar{G}=\sum_{\left\{k_{i}\right\}} G_{i}=G$. Obviously, $n^{s}$ depends on the internal damping rates $\gamma_{10}, \gamma_{21}$ of the quantum dots, the incoherent pumping rate $R$ and the cooperativity strength $G$. Here, $w_{c}=2 /(M G)$ is the standard gain threshold, where the spontaneous emission term in the rate equations of Eq. (44) is neglected [47 49]. Fig. 4 represents

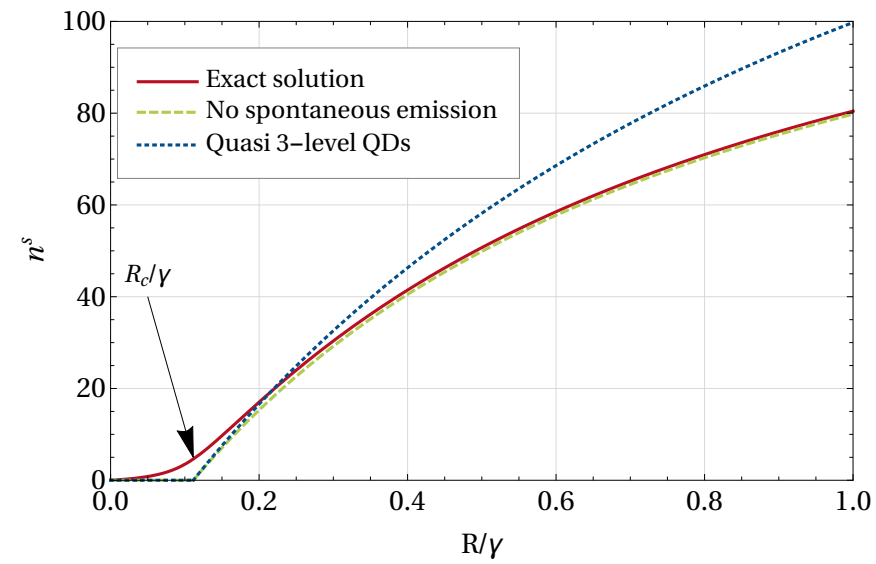

Figure 4: Stationary single-mode $(N=1)$ photon number $n^{s}$ versus scaled pumping rate $R / \gamma$ with $M=10^{3}, \gamma_{10}=\gamma, \gamma_{21}=0.1 \gamma, g=\gamma$ and $\Delta=0$. The red curve shows the analytical solution (46), the green, dashed line represents $n^{s}$ for absent spontaneous emission. The blue, dotted line corresponds to a quasi three-level laser system of Ref. [48] with critical pumping rate $R_{c}=0.111 \gamma$.

the stationary photon number $n^{s}$ versus scaled incoherent pumping rate $R / \gamma$ for the case of a single-mode radiation field which interacts with $M=10^{3}$ equal quantum dots. We chose the internal damping rates $\gamma_{21}=0.1 \gamma$, $\gamma_{10}=\gamma$, the coupling constant $g=\gamma$ and the detuning $\Delta=0$. The red line is the analytical solution specified by Eq. (46). The green, dashed line shows $n^{s}$ in absence of spontaneous emission processes, showing a bifurcation with critical point, $R_{c}=0.111 \gamma$. The blue, dotted curve represents the photon number of the single-mode field interacting with quasi-three level quantum dots [47] and offers the same gain threshold at pumping rate $R_{c}$ as the green line. The comparison between the red and green, dashed curve points out, that in case of the red line the threshold is smeared out and the characteristic amplified spontaneous emission behavior is observable [14, 20, 50]. The blue line highlights an increasing deviation from the red and green line for higher values of $R$.

For increasing detuning $\Delta$, the stationary photon number in terms of pumping rate $R$ offers an increasing gain threshold $R_{c, k}(k=1,2,3,4)$ as demonstrated in Fig. [5 with coupling constant $g=\gamma$, number of quantum dots $M=10^{3}$ and decay rates $\gamma_{21}=0.1 \gamma, \gamma_{10}=\gamma$. The detuning is given by $\Delta=0$ (red line; $R_{c, 1}=0.111 \gamma$ ), $\Delta=10 \gamma$ (green, dashed line; $R_{c, 2}=0.138 \gamma$ ), $\Delta=15 \gamma$ (blue, dotted line; $R_{c, 3}=0.181 \gamma$ ) and $\Delta=20 \gamma$ (orange, dashed-dotted line; $\left.R_{c, 4}=0.271 \gamma\right)$. For decreasing detuning $\Delta$, the slope of the function $n^{s}(R / \gamma)$ increases until the saturation regime is reached.

A similar behavior is observable for $n^{s}(R / \gamma)$ for decreasing coupling $g$ as shown in Fig. [6 with decay rates $\gamma_{21}=0.1 \gamma, \gamma_{10}=\gamma$, detuning $\Delta=0$ and quantum dot number $M=10^{3}$. The coupling constants are chosen to 


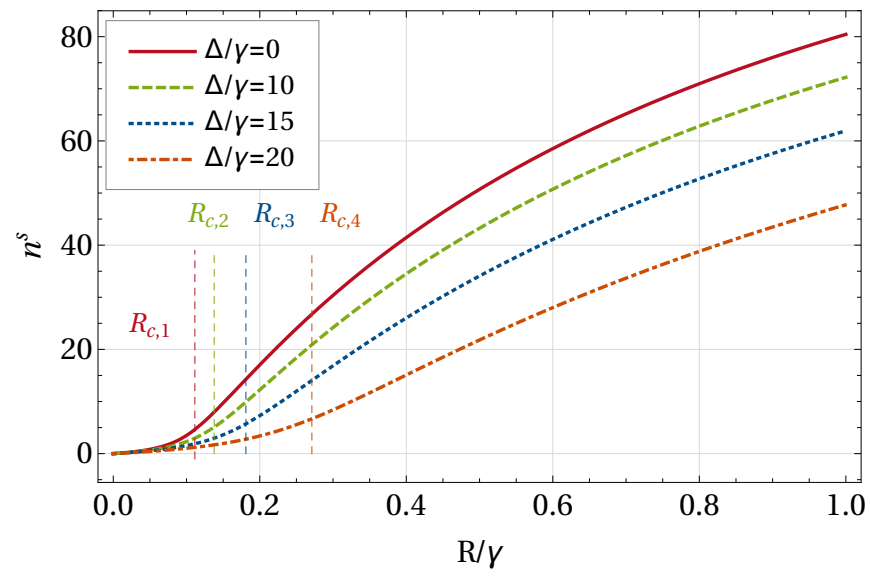

Figure 5: Stationary single-mode $(N=1)$ photon number $n^{s}$ versus scaled pumping rate $R / \gamma$ with parameters $g=\gamma, M=10^{3}, \gamma_{21}=0.1 \gamma, \gamma_{10}=\gamma$ and different detuning $\Delta=0$ (red line; $R_{c, 1}=0.111 \gamma$ ), $\Delta=10 \gamma$ (green, dashed line; $R_{c, 2}=0.138 \gamma$ ), $\Delta=15 \gamma$ (blue, dotted line; $R_{c, 3}=0.181 \gamma$ ) and $\Delta=20 \gamma$ (orange, dashed-dotted line; $R_{c, 4}=0.271 \gamma$ ) as well as corresponding critical pumping rates $R_{c}$.

be $g=0.06 \gamma$ (green line; $R_{c, 1}=0.262 \gamma$ ) and $g=0.1 \gamma$ (blue, dotted-dashed line; $R_{c, 2}=0.145 \gamma$ ), $g=\gamma$ (red, dashed line; $R_{c, 3}=0.111 \gamma$ ). For decreasing coupling constant $g$, the gain threshold tends to higher values of pumping rate $R$.

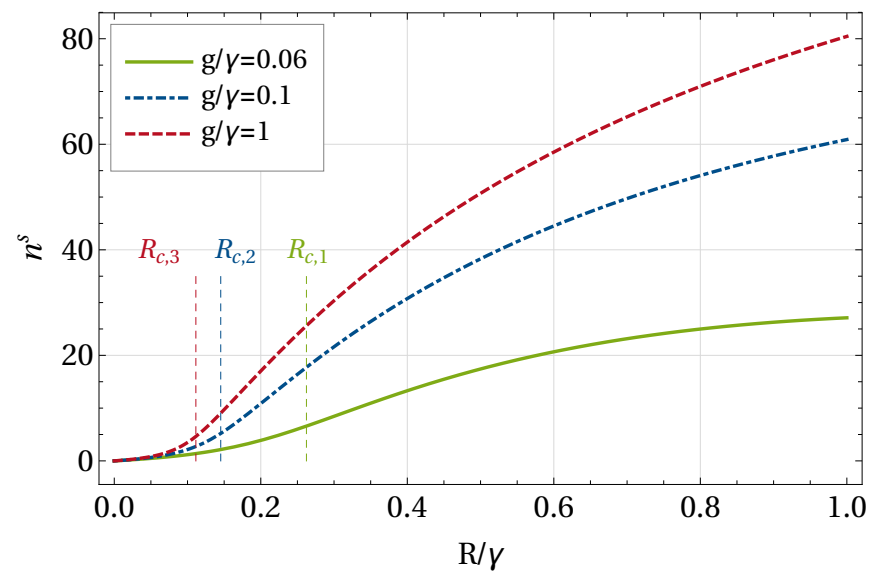

Figure 6: Stationary single-mode $(N=1)$ photon number $n^{s}$ versus scaled pumping rate $R / \gamma$ for vanishing detuning, i.e. $\Delta=0$, parameters $M=10^{3}$, $\gamma_{21}=0.1 \gamma, \gamma_{10}=\gamma$ and varying coupling constant $g=0.06 \gamma$ (green line; $R_{c, 1}=0.262 \gamma$ ), $g=0.1 \gamma$ (blue, dotted-dashed line; $R_{c, 2}=0.145 \gamma$ ) and $g=\gamma$ (red, dashed line; $R_{c, 3}=0.111 \gamma$ ).

\section{Multimode ASE field with identical quantum dots}

For a multimode radiation interacting with equal quantum dots inside the diode system, the rate equations (44) reduce to

$$
\begin{aligned}
\dot{n}_{i}= & M \gamma G_{i}\left(n_{i} w+\sigma_{11}\right)-2 \gamma^{l r} n_{i}+n^{l} \gamma^{l}+n^{r} \gamma^{r} \\
\dot{\sigma}_{22}= & \sum_{\left\{k_{i}\right\}} \gamma G_{i}\left(n_{i} w+\sigma_{11}\right)-\Gamma_{22} \sigma_{22}+\left(R+\gamma_{20}\right) \sigma_{00} \\
& +\gamma_{21}\left(n_{21}+1\right) \sigma_{11} \\
\dot{\sigma}_{11}= & -\sum_{\left\{k_{i}\right\}} \gamma G_{i}\left(n_{i} w+\sigma_{11}\right)+\gamma_{21} n_{21} \sigma_{22}-\Gamma_{11} \sigma_{11} \\
& +\gamma_{10}\left(n_{10}+1\right) \sigma_{00} \\
\dot{\sigma}_{00}= & -\Gamma_{00} \sigma_{00}+\gamma_{10} n_{10} \sigma_{11}+R \sigma_{22} .
\end{aligned}
$$

To find an analytical solution of the stationary photon number $n^{s}$, we introduce an order parameter,

$$
\varphi \equiv \gamma \sum_{\left\{k_{i}\right\}} G_{i} n_{i}^{s}, \quad n^{s}=\sum_{\left\{k_{i}\right\}} n_{i}^{s},
$$

for the diode system in detailed balance [51]. The order parameter corresponds to a weighted stationary total photon number, $n^{s}$. The stationary photon number of mode $i$,

$$
n_{i}^{s}(\varphi)=\frac{\sigma_{11}^{s}(\varphi)}{w_{i}^{c}-w^{s}(\varphi)}, \quad w_{i}^{c}=\frac{2}{M G_{i}},
$$

is a function of $\varphi$ with stationary excited state and inversion,

$$
\begin{aligned}
\sigma_{11}^{s}(\varphi) & =\frac{\varphi\left(\gamma_{10}+R\right)+\gamma_{10} R}{\alpha \varphi+\beta}, \\
w^{s}(\varphi) & =\frac{\gamma_{10} R-\left(\gamma \bar{G}+\gamma_{21}\right)\left(\gamma_{10}+R\right)}{\alpha \varphi+\beta} .
\end{aligned}
$$

$\alpha$ and $\beta$ depend on the incoherent pumping rate $R$ (see Def. (47) and (48)) and $\bar{G}=\sum_{\left\{k_{i}\right\}} G_{i}$ is the sum over all $N$ cooperativity strength, $G_{i}$. Inserting Eq. (51) in Eq. (50), we get a closed relation for $\varphi$,

$$
\varphi=\sum_{\left\{k_{i}\right\}} \frac{\gamma G_{i} \sigma_{11}^{s}(\varphi)}{w_{i}^{c}-w^{s}(\varphi)}=\frac{\sigma_{11}^{s}(\varphi)}{w^{s}(\varphi)}\left[\sum_{\left\{k_{i}\right\}} \frac{\gamma G_{i} w_{i}^{c}}{w_{i}^{c}-w^{s}(\varphi)}-\gamma \bar{G}\right],
$$

whose solution and therefore the photon number itself can be calculated numerically.

In good approximation, we replace $w_{i}^{c}$ in the enumerator of the second term in the brackets of Eq. (54) by the minimum gain threshold $w_{\min }^{c}=2 /\left(M G_{\max }\right)$ with $G_{\max } \equiv G\left(g_{i}=2 / 3 g_{\max }, R\right)$ and maximum coupling con- 
stant $g_{\max }$. Eq. (54) simplifies to

$$
\begin{aligned}
\varphi & \approx-\gamma \bar{G} \frac{\sigma_{11}^{s}(\varphi)}{w^{s}(\varphi)}+w_{\min }^{c} \frac{\varphi}{w^{s}(\varphi)} \\
& =\frac{\gamma_{10} R-\gamma_{21}\left(\gamma_{10}+R\right)-w_{\min }^{c} \beta}{2 w_{\min }^{c} \alpha} \\
& +\sqrt{\left(\frac{\gamma_{10} R-\gamma_{21}\left(\gamma_{10}+R\right)-w_{\min }^{c} \beta}{2 w_{\min }^{c} \alpha}\right)^{2}+\frac{\gamma \bar{G} \gamma_{10} R}{w_{\min }^{c} \alpha}}
\end{aligned}
$$

and we can specify an approximate solution of the stationary intrawaveguide photon number $n^{s}$ (see Eq. (51)) of a QDSLD based on our microscopic theory.

a. Rectangular shape of the cooperativity strength In case of a QDSLD with identical QDs and rectangular distributed cooperativity strength $G_{i}=G$, the resulting rate equations agree with the rate equations of a singlemode QDSLD leading to an exact analytical solution for the stationary photon number $n^{s}=\sum_{\left\{k_{i}\right\}} n_{i}^{s}$ given by Eq. (46).

b. Gaussian shaped coupling Motivated by the observed Gaussian-shaped spectrum (see Fig. 1), Fig. 7 visualizes the stationary photon number $n^{s}$ as a function of $R / \gamma$ for a radiation field composed of $N=30$ modes which interacts with $M=10^{4}$ equal quantum dots with Gaussian coupling constant,

$$
g\left(\Delta_{i}\right)=g_{0} \exp \left(-\frac{\left(\Delta_{i}-\bar{\Delta}\right)^{2}}{2 \sigma^{2}}\right),
$$

where we have chosen an amplitude $g_{0}=\gamma$, expectation value $\bar{\Delta}=0$ and width $\sigma=\gamma$. Damping rates and detuning are $\gamma_{21}=0.1 \gamma, \gamma_{10}=\gamma$ and $\Delta_{i}=i \gamma$. The red line shows the exact, numerically calculated solution of $n^{s}$ and the blue, dashed line is the approximated one, specified by Eq. (50), (51) and (55). The green, dotted line is the solution of $n^{s}$ for negligible spontaneous emission contribution in the sense of Ref. 47] with critical pumping rate, $R_{c}=0.111 \gamma$. Comparison of these three lines points out that the approximate photon number (blue, dashed) and the exact (red) solution agrees well for smaller values of $R$. For $R>0.4 \gamma$, their deviation increases, in contrast to the case of absent spontaneous emission processes approaching the exact solution.

Fig. 8 shows the intrawaveguide photon number $n_{i}^{s}$ of a radiation field with $N=30$ modes as a function of detuning of the $i$ th mode with Gaussian distributed coupling constant, described by Eq. (56), having an amplitude $g_{0}=\gamma$, a mean value $\bar{\Delta} / \gamma=N / 10$ and a standard deviation $\sigma / \gamma=N / 5$. The external damping rates are again $\gamma_{21}=0.1 \gamma$ and $\gamma_{10}=\gamma$. The scaled pumping rate $R / \gamma$ was chosen to be $R / \gamma=0.1,0.3,0.5,1$. The gain medium consists of $M=10^{4}$ quantum dots. This photon number versus frequency is of Gaussian-like shape with nonlinear corrections and exhibits an increasing central frequency and width when increasing the pumping rate $R$. Thus, the photon number is coined by the shape of $G_{i}$.

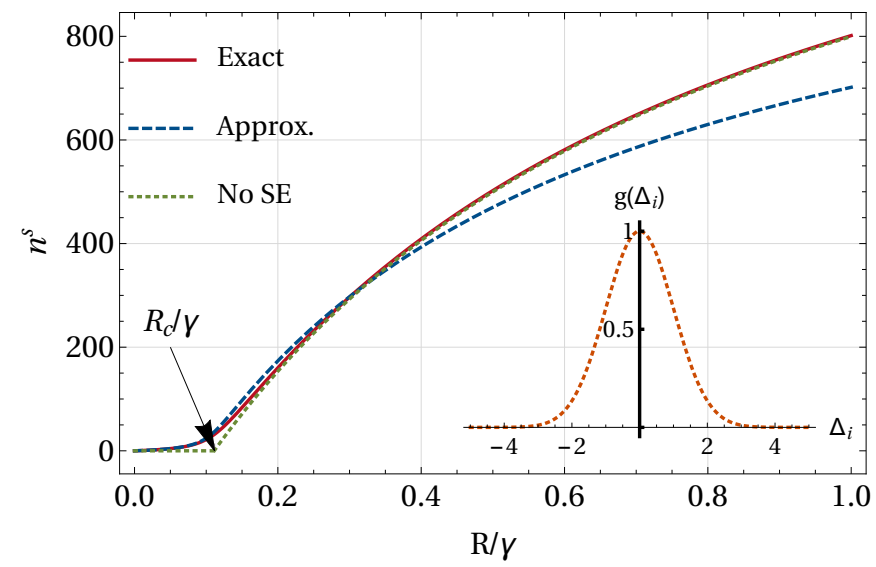

Figure 7: Photon number $n^{s}$ versus scaled incoherent pumping rate $R / \gamma$ for a $N=30$ mode radiation field, interacting with $M=10^{4}$ equal quantum dots. The coupling constant $g_{i}$ is Gaussian-shaped (see Eq. (56)) with $g_{0}=\gamma, \bar{\Delta}=0$ and $\sigma=\gamma$. Decay rates and detuning are $\gamma_{21}=0.1 \gamma, \gamma_{10}=\gamma$ and $\Delta_{i}=i \gamma$. The red line reflects the numerical photon number, the blue, dashed line is the approximated solution described in Eq. (55) and the green, dotted line is the solution for absent spontaneous emission (SE) processes [47] with critical pumping rate $R_{c}=0.111 \gamma$.

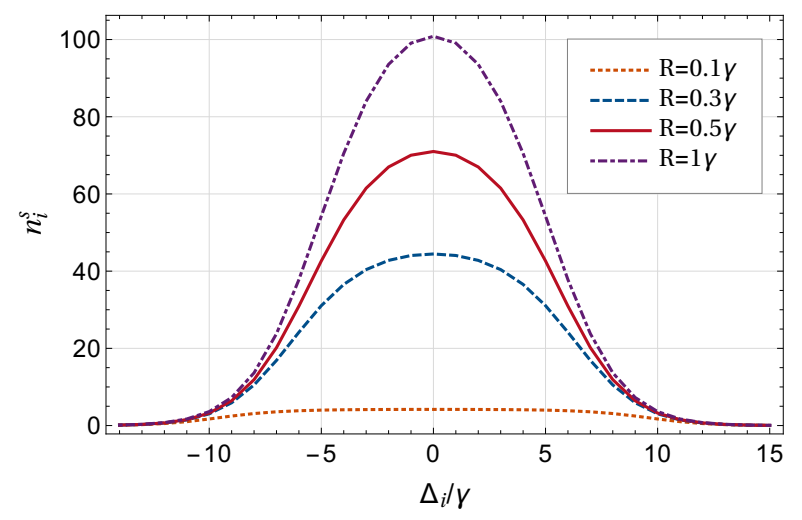

Figure 8: Stationary multimode photon number $n^{s}$ of the radiation field with $N=30$ modes versus scaled detuning $\Delta_{i} / \gamma$ for different scaled pumping rates $R / \gamma=0.1,0.3,0.5,1$. Here, the coupling constants $g_{i}$ are Gaussian distributed (see Eq. (56)) with mean value $\bar{\Delta} / \gamma=N / 10$ and standard deviation $\sigma / \gamma=N / 5$. The damping rates are chosen to be $\gamma_{10}=\gamma, \gamma_{21}=0.1 \gamma$ and the gain medium consists of $M=10^{4}$ quantum dots.

\section{OUTPUT SPECTRUM}

Up to now, we have studied only the intrawaveguide system in terms of rate equations, Eq. (44). To compare our theoretical predictions with the experimental data, we study the optical power spectrum of a QDSLD. The physical problem is sketched in Fig. 9.

By the help of a single detector at fixed position 
$\mathbf{r}_{d}=\left(x_{d}, y_{d}, z_{d}\right)$, the first-order correlation function is determined. In App B, we investigate in more detail the

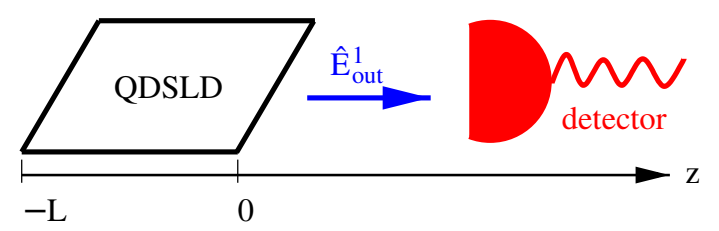

Figure 9: Sketch of the detection process to determine the first-order correlation function of the emitted QDSLD light at position $\mathbf{r}_{\mathrm{d}}$ using a detector with cross-section area $A_{d}$.

spectral density of an empty diode, i.e. without any gain medium. Here, we follow the main ideas and proceedings of App. B and apply them to the case of a truly incoherent QDSLD.

The real output temporal field correlation function at the position of the detector with cross-section area $A_{d}$,

$$
G^{(1)}(\tau)=\lim _{t \rightarrow \infty} \int_{A_{d}} d x_{d} d y_{d}\left\langle\hat{\mathbf{E}}_{\text {out }}^{1(-)}\left(\mathbf{r}_{d}, t\right) \hat{\mathbf{E}}_{\text {out }}^{1(+)}\left(\mathbf{r}_{d}, t+\tau\right)\right\rangle,
$$

is directly connected to the power spectrum according to the Wiener-Khintchine theorem,

$$
S(\omega)=\frac{\mathcal{C}}{\pi} \operatorname{Re} \int_{0}^{\infty} d \tau e^{\mathrm{i} \omega \tau} G^{(1)}(\tau), \quad \mathcal{C}=2 \epsilon_{0} c .
$$

Relying on the concept of the input-output formalism (see App. B) the output operator of channel $\alpha$,

$$
d \hat{b}_{i, \text { out }}^{\alpha}(t)=d \hat{b}_{i, \text { in }}^{\alpha}(t)+\zeta_{i}^{\alpha} \hat{a}_{i}(t) d t
$$

with $\zeta_{i}^{\alpha}$ defined in (26), depends on the input operators of channel $\alpha$ and the internal modes $\hat{a}_{i}$ of the quantized field. According to the orientation of the output facets shown in Fig. 2, channel 1 enters the diode on the right hand side at $z=0$. The electric output field $\hat{\mathbf{E}}_{\text {out }}^{1}$ is assumed to be guided in a single-mode fiber yielding to a radiation field propagating along the $z$-direction. It strikes the detector at $z=z_{d}$.

Evaluating the two-time correlation of the output operators with $t<t^{\prime}=t+\tau$ in consideration of Eq. (599), we find

$$
\begin{aligned}
& \left\langle d \hat{b}_{i, \text { out }}^{1 \dagger}(t) d \hat{b}_{j, \text { out }}^{1}\left(t^{\prime}\right)\right\rangle=\left\langle d \hat{b}_{i, \text { in }}^{1 \dagger}(t) d \hat{b}_{j, \text { in }}^{1}\left(t^{\prime}\right)\right\rangle \\
& +\zeta_{i}^{1 *}\left\langle\hat{a}_{i}^{\dagger}(t) d \hat{b}_{j, \text { in }}^{1}\left(t^{\prime}\right)\right\rangle d t+\zeta_{j}^{1}\left\langle d \hat{b}_{i, \text { in }}^{1 \dagger}(t) \hat{a}_{j}\left(t^{\prime}\right)\right\rangle d t^{\prime} \\
& +\zeta_{i}^{1 *} \zeta_{j}^{1}\left\langle\hat{a}_{i}^{\dagger}(t) \hat{a}_{j}\left(t^{\prime}\right)\right\rangle d t d t^{\prime} .
\end{aligned}
$$

In the following, we choose vacuum as an input, where the first three terms in Eq. (60) vanish. Thus, the output field is directly related to the first-order autocorrelation function of the ASE field modes at different time events $t<t^{\prime}$,

$$
\begin{aligned}
d\left\langle\hat{a}_{i}^{\dagger}(t) \hat{a}_{s}\left(t^{\prime}\right)\right\rangle= & -\mathrm{i}\left(\Delta_{s}-\mathrm{i} \gamma^{l r}\right)\left\langle\hat{a}_{i}^{\dagger}(t) \hat{a}_{s}\left(t^{\prime}\right)\right\rangle d t^{\prime} \\
& +\sum_{j=1}^{M} g_{s}^{j *}\left\langle\hat{a}_{i}^{\dagger}(t) \hat{\sigma}_{21}^{j}\left(t^{\prime}\right)\right\rangle d t^{\prime} .
\end{aligned}
$$

Applying again the decorrelation approximation to $\left\langle\hat{a}_{i}^{\dagger}(t) \hat{\sigma}_{21}^{j}\left(t^{\prime}\right)\right\rangle$ (see Eq. (43)) and adiabatic elimination, Eq. (61) reduces to

$$
\begin{array}{r}
d\left\langle\hat{a}_{i}^{\dagger}(t) \hat{a}_{s}\left(t^{\prime}\right)\right\rangle=-\left(\eta_{s}\left(t^{\prime}\right)+\mathrm{i} \chi_{s}\left(t^{\prime}\right)\right)\left\langle\hat{a}_{i}^{\dagger}(t) \hat{a}_{s}\left(t^{\prime}\right)\right\rangle d t^{\prime} \\
+\sum_{j=1}^{M} \sum_{\substack{\left\{k_{m}\right\} \\
m \neq s}} \frac{g_{m}^{j} g_{s}^{j *} w^{j}\left(t^{\prime}\right)}{\Gamma_{21}^{j}+\mathrm{i} \delta \omega_{12}^{j}} \times\left\langle\hat{a}_{i}^{\dagger}(t) \hat{a}_{m}\left(t^{\prime}\right)\right\rangle d t^{\prime}
\end{array}
$$

with

$$
\chi_{i}(t)-\mathrm{i} \eta_{i}(t)=\Delta_{i}-\mathrm{i} \gamma^{l r}+\sum_{j=1}^{M} \frac{\left|g_{i}^{j}\right|^{2} w^{j}(t)}{\delta \omega_{12}^{j}-\mathrm{i} \Gamma_{21}^{j}} .
$$

We claim that off-diagonal elements of the coupling matrix are small in comparison to the diagonal one. Furthermore, in first-order perturbation theory we disregard the last term of Eq. (62).

In equilibrium, the inversion tends to a constant value, $\lim _{t \rightarrow \infty}\left\langle\hat{w}^{j}(t+\tau)\right\rangle=w^{j s}=$ const.. Clearly, as we want to investigate the stationary power spectrum of a QDSLD (58), the time dependent inversion arising in the expressions of $\eta_{s}\left(t^{\prime}\right)$ and $\chi_{s}\left(t^{\prime}\right)$ of Eq. (62) can be replaced by $w^{j s}$. For small QD transition deviations $\delta \omega_{12}^{j}$, we can replace propagation and absorption coefficients [52] according to

$$
\chi_{i} \approx \Delta_{i}, \quad \eta_{i} \approx \gamma^{l r}-\xi_{i}, \quad \xi_{i}=2 \sum_{j=1}^{M} \frac{\left|g_{i}^{j}\right|^{2} w^{j s}}{\Gamma_{21}^{j}},
$$

and the field correlation function with $t^{\prime}>t$ reads

$$
\left\langle\hat{a}_{i}^{\dagger}(t) \hat{a}_{j}\left(t^{\prime}\right)\right\rangle=n_{i}(t) e^{-\mathrm{i}\left(\chi_{i}-\mathrm{i} \eta_{i}\right)\left(t^{\prime}-t\right)} \delta_{i j} .
$$

Finally, the correlation function for the output operators $d \hat{b}_{i, \text { out }}^{1}$ at different time events $t, t^{\prime}$ reads

$$
\left\langle d \hat{b}_{i, \text { out }}^{1 \dagger}(t) d \hat{b}_{j, \text { out }}^{1}\left(t^{\prime}\right)\right\rangle=\frac{\gamma^{r}}{2}\left\langle\hat{a}_{i}^{\dagger}(t) \hat{a}_{j}\left(t^{\prime}\right)\right\rangle \delta_{i j} d t d t^{\prime} .
$$

The stationary optical power spectrum in the original Heisenberg picture (20) is given by

$$
S(\omega)=\sum_{\left\{k_{i}>0\right\}} P_{i} \mathcal{L}_{\Gamma_{i}}\left(\omega-\omega_{i}\right) n_{i}^{s} \quad P_{i}=\frac{\hbar \omega_{i} c \gamma^{r}}{L \Delta \omega_{i}^{r}}
$$

with optical power $P_{i}$ and Lorentzian function

$$
\mathcal{L}_{\Gamma}(\omega)=\frac{1}{\pi} \frac{\Gamma}{(\Gamma / 2)^{2}+\omega^{2}}, \quad \Gamma=2\left(\gamma^{l r}-\xi\right),
$$


with $\int_{-\infty}^{\infty} d \omega \mathcal{L}_{\Gamma}(\omega)=1$. Assuming negligible small deviation of $\omega_{i}$ from a central frequency $\bar{\omega}$ as well as equidistant frequency separations, i.e. $\Delta \omega_{i}^{r}=\Delta \omega^{r}$, the continuous optical power spectrum

$$
S(\omega)=\frac{P}{\Delta \omega^{r}} \int_{-\infty}^{\infty} d \omega^{\prime} \mathcal{L}_{\Gamma}\left(\omega-\omega^{\prime}\right) n^{s}\left(\omega^{\prime}\right), \quad P=\frac{\hbar \bar{\omega} c \gamma^{r}}{L \Delta \omega^{r}}
$$

is a convolution of a Lorentzian curve with the stationary intrawaveguide photon number specified by the rate equations (44).

Fig. 10 visualizes the spectral density as a function of angular frequency. The black dots are the experimental data and the green line is a Gaussian fit, both already depicted in Fig. 1. The red, dashed curve reflects the so-

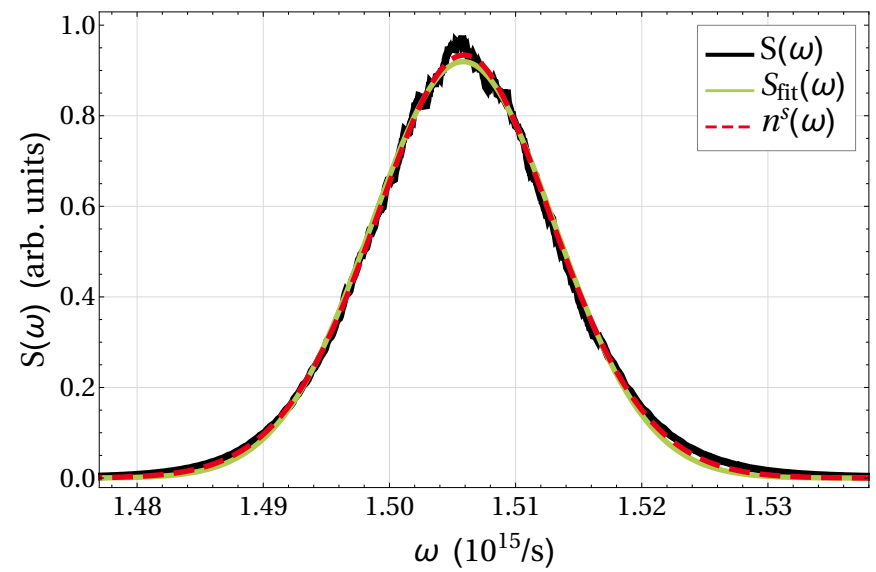

Figure 10: Experimental power spectrum $S(\omega)$ (black) and Gaussian fit (green line) (see Fig. 1) and stationary intrawaveguide photon number $n^{s}(\omega)$ (red, dashed line) for $M=10^{4}$ identical QDs and Gaussian shaped $G(\omega)$ (see Eq. (C2) ) with fit parameters listed in Tab. I.

lution of our quantum theory of light-emitting QDSLDs. More precisely, it shows the intrawaveguide photon number in case of $M=10^{4}$ equal quantum dots and Gaussian shaped cooperativity strength (see Eq. (C2)). Values of pumping rate and internal damping rates are again chosen to be $R=0.5 \gamma, \gamma_{10}=\gamma$ and $\gamma_{21}=0.1 \gamma$. Practically, the numerical solution of $n^{s}(\omega)$ is investigated by solving the rate equations and using Newton's method. Both, Gaussian fit and numerical solution of the internal photon number fit the data remarkably well revealing a power spectrum with a $\mathrm{THz}$ spectral width. Thus, the optical spectrum of a QDSLD is primarily affected by the stationary intrawaveguide photon number itself. Its shape again depends significantly on the distribution of the cooperativity strength $G(\omega)$. Knowing all experimental parameters, we are able to replicate each measured optical power spectrum of a QDSLD from microscopic considerations. Furthermore, knowing the experimental data, we can specify unknown, relevant parameters like coupling strength between QD and ASE field and so on.

\section{CONCLUSION}

In this article, we have studied the amplified spontaneous emission of light-emitting quantum dot superluminescent diodes in terms of first-order temporal autocorrelation function. We presented a microscopic theory of QDSLDs, which considers the specific gain medium, formed by an inhomogeneous ensemble of QDs, as well as tilted end facets. The former was modeled by distinguishable three-level systems, incoherently pumped and interacting with a multimode radiation field, which couples to the outside by two beam splitters enclosing the active medium. We derived quantum stochastic differential equations in the quantum white noise limit, which allow to calculate first- as well as higher-order temporal correlation functions.

The resulting rate equations for the optical power densities and level occupations of the quantum dots within the QDSLD were studied for the limiting cases of a singlemode and a multimode ASE radiation field interacting with $M$ equal quantum dots. We showed, that the stationary intrawaveguide photon number as a function of the incoherent pumping rate agrees well with experimental results, where the typical amplified spontaneous emission transition is visible.

We have applied the input-output formalism to determine the optical power spectrum at the detector's position. As a main result, we showed that this spectral density is a convolution of a Lorentzian curve with the intrawaveguide photon number, whose shape is coined by the shape of the cooperativity strength.

\section{ACKNOWLEDGMENT}

We like to thank Sébastien Blumenstein (name of birth Hartmann) for the experimental data, all experimental details and fruitful discussions. Furthermore, we thank the German Aerospace Center (DLR) (50 WM 1557) and the Deutsche Forschungsgemeinschaft (DLR) for financial support.

\section{Appendix A: Quantum Ito calculus}

One can analyze the dynamics of Heisenberg operators $\hat{Y}(t)$ and $\left\{\hat{X}_{m}(t)\right\}$ of a small open quantum system coupled to $\mathcal{M}$ reservoirs by the quantum stochastic differential equation (QSDE)

$$
\begin{aligned}
d \hat{Y}= & -\frac{i}{\hbar}\left[\hat{Y}, \hat{H}_{s}\right] d t+\sum_{m=1}^{\mathcal{M}}\{ \\
& \gamma_{m}\left(X_{m}^{\dagger}\left[\hat{Y}, \hat{X}_{m}\right]-\left[\hat{Y}, \hat{X}_{m}^{\dagger}\right] \hat{X}_{m}\right)\left(n_{m}+1\right) d t \\
& +\gamma_{m}\left(-\left[\hat{Y}, \hat{X}_{m}\right] \hat{X}_{m}^{\dagger}+\hat{X}_{m}\left[\hat{Y}, \hat{X}_{m}^{\dagger}\right]\right) n_{m} d t \\
& \left.+d \hat{B}_{m}^{\dagger}(t)\left[\hat{Y}, \hat{X}_{m}\right]-\left[\hat{Y}, \hat{X}_{m}^{\dagger}\right] d \hat{B}_{m}(t)\right\}
\end{aligned}
$$


in the Ito interpretation [33, 40]. The stochastic increments $d \hat{B}_{m}(t)=\sqrt{\gamma_{m}} e^{-\mathrm{i} \phi_{m}} d \hat{b}_{m}(t)$ are proportional to the Ito quantum noise increments $d \hat{b}_{m}$. In the meansquare limit, one can postulate infinitesimal relations for Wiener noise increments

$$
\begin{aligned}
& d \hat{b}_{m}(t) d \hat{b}_{m}^{\dagger}(t)=\left(n_{m}+1\right) d t \\
& d \hat{b}_{m}^{\dagger}(t) d \hat{b}_{m}(t)=n_{m} d t, \\
& d \hat{b}_{m}(t) d \hat{b}_{m}(t)=0 .
\end{aligned}
$$

The heat content of the $m$ unsqueezed reservoirs is proportional to the occupation numbers $n_{m}$. Time evolution according to Eq. A1 is consistent with the Ito rule of calculus

$$
d(\hat{X} \hat{Y}) \equiv \hat{X} d \hat{Y}+(d \hat{X}) \hat{Y}+d \hat{X} d \hat{Y}
$$

\section{Appendix B: Multi-channel transmission spectrum of a passive waveguide}

We analyze the output field measured by the single photon detector at position $\mathbf{r}_{d}=\left(x_{d}, y_{d}, z_{d}\right)$ under consideration of the facet geometry. For simplicity, we study the case of an empty diode, that is in absent of any quantum dot or similar gain medium. Fig. 11 illustrates the empty diode with the two beam splitters modeling the facets, where electrical fields $\hat{\mathbf{E}}^{\alpha}(\mathbf{r}, t)$, enumerated by the channel numbers $\alpha=1, \ldots, 6$, partially entering and simultaneously leaving the diode. The positive frequency

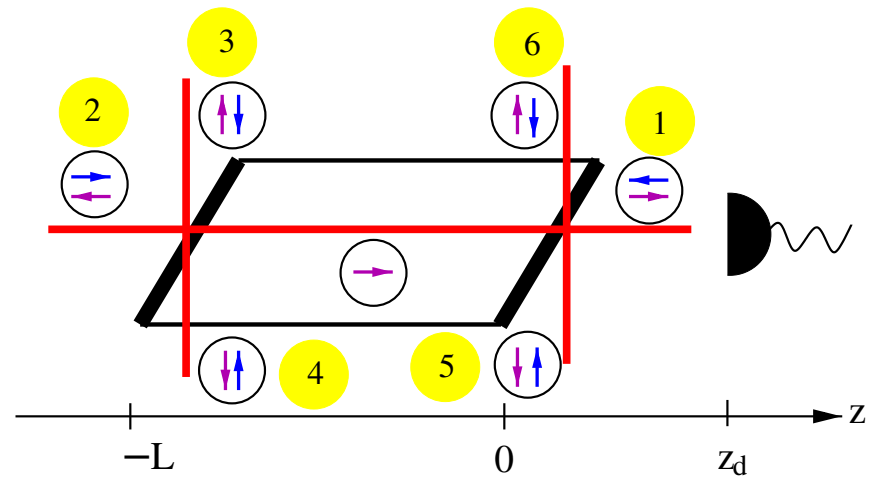

Figure 11: Sketch of the single channels, labeled by numbers between 1 and 6 , striking the output facets of the QDSLD from the left and right hand side.

part of the electric field of channel 1 with $z>0$ was already introduced and is given by Eq. (12).

a. Input-output formalism We apply the inputoutput formalism [33] in order to specify the power spectrum of the diode system. The total Hamiltonian depends on the field Hamiltonian of the ASE field (2) the field Hamiltonian of the reservoir corresponding to each channel (5) and the Hamiltonian describing the interac- tion of the external fields with the ASE radiation field,

$$
\hat{H}_{I}=\sum_{\alpha=1}^{6} \sum_{\left\{k_{i}\right\}} \sum_{q \in M_{i}^{\alpha}} \mathrm{i} \hbar \kappa_{i q}^{\alpha} \hat{b}_{i q}^{\alpha \dagger} \hat{a}_{i}+\text { h.c.. }
$$

The coupling constant $\kappa_{i q}^{\alpha}=\left|\kappa_{i q}^{\alpha}\right| \exp \left(\mathrm{i} \phi_{i}^{\alpha}\right)$ characterizes the coupling strength between the multimode ASE field inside the gain medium and the electrical field channels. The Heisenberg equations of motion of the system modes reads

$$
\begin{aligned}
\dot{\hat{b}}_{i q}^{\alpha} & =-\mathrm{i} \omega_{q} \hat{b}_{i q}^{\alpha}+\kappa_{i q}^{\alpha} \hat{a}_{i}, \\
\dot{\hat{a}}_{i} & =-\mathrm{i} \omega_{i} \hat{a}_{i}-\sum_{\alpha=1}^{6} \sum_{q \in M_{i}^{\alpha}} \kappa_{i q}^{\alpha *} \hat{b}_{i q}^{\alpha} .
\end{aligned}
$$

According to Fig. 11, channel 3 and 5 do not enter the waveguide and can be disregarded. By integrating Eq. (B2) subject to the initial conditions $\hat{b}_{i q 0}^{\alpha}=\hat{b}_{i q}^{\alpha}\left(t_{0}\right)$, one obtains

$$
\begin{gathered}
\hat{b}_{i q}^{\alpha}(t)=e^{-\mathrm{i} \omega_{q}\left(t-t_{0}\right)} \hat{b}_{i q 0}^{\alpha}+\kappa_{i q}^{\alpha} \int_{t_{0}}^{t} d \tau e^{-\mathrm{i} \omega_{q}(t-\tau)} \hat{a}_{i}(\tau), \\
\dot{\hat{a}}_{i}=-\mathrm{i} \omega_{i} \hat{a}_{i}-\sum_{\alpha=1}^{6} \int_{t_{0}}^{t} d \tau K_{i}^{\alpha}(t-\tau) \hat{a}_{i}(\tau)+\hat{f}_{i}^{\alpha}(t) .
\end{gathered}
$$

This procedure defines stochastic Langevin forces $\hat{f}_{i}^{\alpha}$ and memory kernels $K_{i}^{\alpha}$ by

$$
\begin{aligned}
\hat{f}_{i}^{\alpha}(t) & \equiv \sum_{q \in M_{i}^{\alpha}} e^{-\mathrm{i} \omega_{q}\left(t-t_{0}\right)} \kappa_{i q}^{\alpha *} \hat{b}_{i q 0}^{\alpha}, \\
K_{i}^{\alpha}(\tau) & \equiv \sum_{q \in M_{i}^{\alpha}} e^{-\mathrm{i} \omega_{q} \tau}\left|\kappa_{i q}^{\alpha}\right|^{2} .
\end{aligned}
$$

Only for a short time, the stochastic forces are correlated and satisfy a two-time fluctuation-dissipation relation

$$
\left[\hat{f}_{i}^{\alpha}(t), \hat{f}_{j}^{\beta \dagger}\left(t^{\prime}\right)\right]=K_{i}^{\alpha}\left(t-t^{\prime}\right) \delta_{\alpha \beta} \delta_{i j} .
$$

In the Markov limit, one can approximate convolutions of memory kernels with system operators $\hat{c}(t)$ as

$$
\int_{t_{0}}^{t} d \tau K_{i}^{\alpha}(t-\tau) \hat{c}(\tau) \approx\left(\frac{\gamma_{i}^{\alpha}}{2}+\mathrm{i} \delta \omega_{i}^{\alpha}\right) \hat{c}(t),
$$

for times much larger than the correlation time $\tau_{c}$, i.e. $t \gg \tau_{c}$. Now, $\gamma_{i}^{\alpha}$ defines a decay rate and $\delta \omega_{i}^{\alpha}$ is a line shift. Such self-energies implicitly renormalize the bare energies and are not considered furthermore.

The two-time commutation relation of the input field

$$
\begin{aligned}
{\left[\hat{b}_{i, \text { in }}^{\alpha}(t), \hat{b}_{j, \text { in }}^{\beta \dagger}\left(t^{\prime}\right)\right] } & =\delta_{\alpha \beta} \delta_{i j} \delta\left(t-t^{\prime}\right), \\
\delta(\tau) & =\sum_{q \in M_{i}^{\alpha}} \frac{\Delta \omega_{i}}{2 \pi} e^{-i \omega_{q} \tau},
\end{aligned}
$$


has a very short correlation time analog to the Markov approximation (B9). In the same order of approximation, the Langevin force,

$$
\hat{f}_{i}^{\alpha}(t)=\zeta_{i}^{\alpha *} \hat{b}_{i, \text { in }}^{\alpha}(t)
$$

with $\zeta_{i}^{\alpha}$ defined in Eq. (26), is proportional to the input operator. Putting all together, we find the equation of motion of the ASE field mode $i$,

$$
\dot{\hat{a}}_{i}=-\mathrm{i} \vartheta_{i} \hat{a}_{i}-\sum_{\alpha=1}^{6} \hat{f}_{i}^{\alpha}(t), \quad \vartheta_{i}=\omega_{i}-\mathrm{i} \sum_{\alpha=1}^{6} \frac{\gamma_{i}^{\alpha}}{2}
$$

in terms of the input operators and damping rates (28). The corresponding phases are given by Eq. (29), respectively.

Analog calculations in terms of final condition $t_{f}>t$ lead to a similar expression for the ASE field mode,

$$
\dot{\hat{a}}_{i}=-\mathrm{i} \vartheta_{i}^{*} \hat{a}_{i}-\sum_{\alpha=1}^{6} \zeta_{i}^{\alpha *} \hat{b}_{i, \text { out }}^{\alpha} .
$$

Comparison between Eq. (B12) and Eq. (B13) ends up in the fundamental relation,

$$
\hat{b}_{i, \text { out }}^{\alpha}(t)=\hat{b}_{i, \text { in }}^{\alpha}(t)+\zeta_{i}^{\alpha} \hat{a}_{i}(t),
$$

between the input- and output operators.

b. Output spectrum The output spectrum recorded by a single-photon sensitive detector with cross-section area $A$ corresponds to the expectation value,

$$
S_{\text {out }}(\omega)=\mathcal{C}\left\langle\hat{E}^{(-)}(\omega) \hat{E}^{(+)}(\omega)\right\rangle, \quad \mathcal{C}=2 \epsilon_{0} c,
$$

of the system's output operators in frequency space. The components of the power spectrum matrix

$$
\begin{aligned}
S_{\text {out }}(\omega) & =\sum_{\left\{k_{i}, k_{j}>0\right\}} \Upsilon_{i j}\left\langle\hat{b}_{i, \text { out }}^{\alpha \dagger}(\omega) \hat{b}_{j, \text { out }}^{\beta}(\omega)\right\rangle, \\
\Upsilon_{i j} & =\frac{2 \hbar \pi c}{L} \sqrt{\frac{\omega_{i} \omega_{j}}{\Delta \omega_{i} \Delta \omega_{j}}},
\end{aligned}
$$

depend on the components of $\hat{b}_{i, \text { out }}(\omega)$ which again can be calculated by Fourier transforming the input-output relation (B14) under consideration of the Langevin equation Eq. (B13),

$$
\hat{\mathbf{b}}_{i, \text { out }}(\omega)=M_{i}(\omega) \hat{\mathbf{b}}_{i, \text { in }}(\omega),
$$

with input vector $\hat{\mathbf{b}}_{i, \text { in/out }}=\left(\hat{b}_{i, \text { in } / \text { out }}^{1} \ldots \hat{b}_{i, \text { in/out }}^{6}\right)$. The matrix,

$$
M_{i}=\frac{1}{\lambda_{i}}\left(\begin{array}{cccccc}
m_{i, 11} & m_{i, 12} & 0 & m_{i, 14} & 0 & m_{i, 16} \\
m_{i, 21} & m_{i, 22} & 0 & m_{i, 24} & 0 & m_{i, 26} \\
0 & 0 & m_{i, 33} & 0 & 0 & 0 \\
m_{i, 41} & m_{i, 42} & 0 & m_{i, 44} & 0 & m_{i, 46} \\
0 & 0 & 0 & 0 & m_{i, 55} & 0 \\
m_{i, 61} & m_{i, 62} & 0 & m_{i, 64} & 0 & m_{i, 66}
\end{array}\right)
$$

with $\lambda_{i}=\mathrm{i}\left(\omega-\omega_{i}\right)-\gamma_{i}^{l r}$ and elements

$$
m_{i, \alpha \beta}= \begin{cases}\mathrm{i}\left(\omega-\omega_{i}\right)+\frac{1}{2}\left(\gamma_{i}^{\alpha}-\sum_{\alpha^{\prime} \neq \alpha} \gamma_{i}^{\alpha^{\prime}}\right) & \text { for } \alpha=\beta \\ \zeta_{i}^{\alpha} \zeta_{i}^{\beta *} & \text { for } \alpha \neq \beta\end{cases}
$$

satisfies the relation $M_{i}^{\dagger} M_{i}=1$. As depicted in Fig. 11, only the electrical output field of channel 1 contributes to the power spectrum of the diode, i.e. $S(\omega)=\left(S_{\text {out }}(\omega)\right)_{11}$. Clearly, $S(\omega)$ depends significantly on the choice of the input field: it is the convolution of the input field with the response function of the beam splitters.

c. White noise input Consider the case of vacuum input channel fields $1-5$ and a quantum white noise channel 6 with equal damping rates, $\gamma_{i}^{\alpha}=\gamma$. The input matrix components reduce to $\left(S_{i j \text {,in }}\right)_{\alpha \beta}=n^{\alpha} \delta_{\alpha \beta} \delta_{i j}$ with $n^{6}=n=$ const. and $n^{\alpha}=0$ else, and the power spectrum measured by the detector,

$$
S(\omega)=\sum_{\left\{k_{i}>0\right\}} P_{i} \mathcal{L}_{\Gamma}\left(\omega-\omega_{i}\right) n, \quad P_{i}=\frac{\hbar \pi^{2} c \gamma \omega_{i}}{2 \Delta \omega_{i} L}
$$

with $\Gamma=4 \gamma$ is given by the sums of Lorentzians with maximum value at $\omega=\omega_{i}$ and full width at half maximum $\sqrt{2} \gamma$. Assuming that $\omega_{i}$ in the enumerator of power $P_{i}$ differs only slightly from a mean value $\bar{\omega}$, the continuous power spectrum is approximately specified by

$$
S(\omega)=\frac{P}{\Delta \omega} \int_{-\infty}^{\infty} d \tilde{\omega} \mathcal{L}_{\Gamma}(\omega-\tilde{\omega}) n .
$$

\section{Appendix C: Fitting the optical power spectrum}

The smooth Gaussian interpolation of the optical power spectrum $S(\omega)$, which is depicted in Fig. 1, reads

$$
S_{\text {fit }}(\omega)=\frac{S_{0}}{\sqrt{2 \pi} \sigma} e^{-\frac{(\omega-\bar{\omega})^{2}}{2 \sigma^{2}}}, \quad \int_{-\infty}^{\infty} d \omega S_{\text {fit }}(\omega)=S_{0},
$$

with amplitude $S_{0}$, standard deviation $\sigma$ and central frequency $\bar{\omega}$. More generally, one can also propose a Gaussian profile for the cooperativity strength,

$$
G(\omega)=G_{0} e^{-\frac{(\omega-\bar{\omega})^{2}}{2 \sigma^{2}}}
$$

occurring in the quantum model for the intrawaveguide photon number of the QDSLD presented in Sec. III. Other parameters, included in this multimode theory are assumed to be well known and given by $R=0.5 \gamma$, $\gamma_{10}=\gamma, \gamma_{21}=0.1 \gamma$. A comparison of the Gaussian fit of the power spectrum together with the solution of the intrawaveguide photon number with Gaussian distributed cooperativity strength and equal QDs is depicted Fig.10. The obtained fit parameters are listed in Tab. [I. 


\begin{tabular}{c||c|c|c|c} 
Quantity & $\bar{\omega}\left(10^{15} / \mathrm{s}\right)$ & $\sigma\left(10^{12} / \mathrm{s}\right)$ & $G_{0}$ (a.u.) & $S_{0}$ (a.u.) \\
\hline \hline Fit $S_{\text {fit }}(\omega)$ & 1.506 & 7.286 & - & 0.117 \\
\hline Model $G(\omega)$ & 1.506 & 7.962 & $2.467 \times 10^{-4}$ & -
\end{tabular}

Table I: Fit parameters for the Gaussian and the QDSLD model fit corresponding to the power spectrum depicted in Figs. 11 and 10. The normalized sum of the squared residuals are $\sum_{\left\{k_{i}\right\}} r_{i}^{2} / N=1.137 \times 10^{-4}$

(Gauss) and $\sum_{\left\{k_{i}\right\}} r_{i}^{2} / N=0.526 \times 10^{-4}(\mathrm{QDSLD}$ model).

[1] P. Urquhart, O. G. Lopez, G. Boyen, and A. Bruckmann, Int. Signal Processing , 1 (2007).

[2] C. Velez, L. Occhi, and M. B. Raschle, Phot. Spectra 39 (2005)

[3] D. Huang, E. A. Swanson, C. P. Lin, J. S. Schuman, W. G. Stinson, W. Chang, M. R. Hee, T. Flotte, K. Gregory, C. A. Puliafito, and J. G. Fujimoto, Science 254, 1178 (1991)

[4] P. Judson, K. Groom, D. Childs, M. Hopkinson, N. Krstajic, and R. Hogg, Microelectron. J. 40, 588 (2009) workshop of Recent Advances on Low Dimensional Structures and Devices (WRA-LDSD).

[5] A. E. Siegman, Lasers (Univ. Science Books, Mill Valley, 1986).

[6] D. S. Wiersma, Nat. Phys. 4, 359 (2008).

[7] T. B. Pittman, Y. H. Shih, D. V. Strekalov, and A. V. Sergienko, Phys. Rev. A 52, R3429 (1995).

[8] S. Kuhn, S. Hartmann, and W. Elsäßer, Opt. Lett. 41, 2863 (2016)

[9] S. Hartmann, A. Molitor, and W. Elsäßer, Opt. Lett. 40, 5770 (2015)

[10] S. Hartmann and W. Elsäßer, Sci. Rep. 7, 41866 (2017)

[11] F. Boitier, A. Godard, A. Ryasnyanskiy, N. Dubreuil, P. Delaye, C. Fabre, and E. Rosencher, Opt. Express 18, 20401 (2010).

[12] F. Boitier, A. Godard, N. Dubreuil, P. Delaye, C. Fabre, and R. Rosencher, Nat. Comm. 2 (2011)

[13] M. Blazek, S. Hartmann, A. Molitor, and W. Elsäßer, Opt. Lett. 36, 3455 (2011)

[14] M. Blazek and W. Elsäßer, IEEE J. Quantum Electron. 48, 1578 (2012).

[15] F. Boitier, A. Godard, N. Dubreuil, P. Delaye, C. Fabre, and E. Rosencher, Phys. Rev. A 87, 013844 (2013).

[16] M. Blazek and W. Elsäßer, Phys. Rev. A 84, 063840 (2011)

[17] F. Boitier, A. Godard, E. Rosencher, and C. Fabre, Nat. Phys. 5, 267 (2009)

[18] G. I. Peters and L. Allen, J. Phys. A 4, 238 (1971).

[19] L. Allen and G. I. Peters, J. Phys. A 4, 377 (1971)

[20] L. Allen and G. I. Peters, J. Phys. A 4, 564 (1971)

[21] G. I. Peters and L. Allen, J. Phys. A 5, 546 (1972)

[22] A. Jechow, M. Seefeldt, H. Kurzke, A. Heuer, and R. Menzel, Nat. Photon. 7, 973 (2013).

[23] J. Kiethe, A. Heuer, and A. Jechow, Laser Phys. Lett. 14, 086201 (2017)
[24] A. Uskov, T. Berg, and J. Mørk, IEEE J. Quantum Electron. 40, 306 (2004).

[25] P. Bardella, M. Rossetti, and I. Montrosset, IEEE J. Sel. Top. Quant. 15, 785 (2009).

[26] M. Rossetti, P. Bardella, and I. Montrosset, IEEE J. Quantum Electron. 47, 139 (2011).

[27] Z. Q. Li and Z. M. S. Li, IEEE J. Quantum Electron. 46, 454 (2010).

[28] M. Van der Poel, E. Gehrig, O. Hess, D. Birkedal, and J. M. Hvam, IEEE J. Quantum Electron. 41, 1115 (2005)

[29] M. Wegert, N. Majer, K. Lüdge, S. Dommers-Völkel, J. Gomis-Bresco, A. Knorr, U. Woggon, and E. Schöll, Semicond. Sci. Tech. 26, 014008 (2011)

[30] S. Hartmann, F. Friedrich, A. Molitor, M. Reichert, W. Elsäßer, and R. Walser, NJP 17, 043039 (2015)

[31] S. Hartmann, A. Molitor, M. Blazek, and W. Elsäßer, Opt. Lett. 38, 1334 (2013)

[32] M. Sargent, M. Scully, and W. Lamb, Laser physics, Bd. 201067196 (Addison-Wesley Pub. Co., 1976).

[33] C. Gardiner and P. Zoller, Quantum noise (Springer, 2000).

[34] T. Lee, J. C. A. Burrus, and B. I. Miller, IEEE J. Quantum Electron. QE-9, 820 (1973).

[35] M. C. Amann and J. Boeck, Electron. Lett. 15, 41 (1979)

[36] We thank Sébastien Blumenstein (name of birth Hartmann) for providing the experimental data.

[37] G. A. Alphonse, D. B. Gilbert, M. G. Harvey, and M. Ettenberg, IEEE J. Quantum Electron. 24, 2454 (1988)

[38] M. Blazek and W. Elsässer, Private communication.

[39] Z. Zhang, I. Luxmoore, C. Jin, H. Liu, Q. Jiang, K. Groom, D. Childs, M. Hopkinson, A. Cullis, and R. Hogg, Appl. Phys. Lett. 91, 081112 (2007).

[40] C. W. Gardiner and M. J. Collett, Phys. Rev. A 31, 3761 (1985).

[41] P. Michler, A. Imamoglu, M. D. Mason, P. J. Carson, G. F. Strouse, and S. K. Buratto, Nature 406, 968 (2000).

[42] M. J. Collett and C. W. Gardiner, Phys. Rev. A 30, 1386 (1984).

[43] L. Tian and H. J. Carmichael, Quantum Opt. 4, 131 (1992)

[44] T. Tawara, H. Kamada, T. Tanabe, T. Sogawa, H. Okamoto, P. Yao, P. K. Pathak, and S. Hughes, Opt. Express 18, 2719 (2010) 
[45] M. R. Sturm, B. Rein, T. Walther, and R. Walser, J. Opt. Soc. Am. B 31, 1964 (2014).

[46] S. Barnett and P. Methods in Theoretical Quantum Optics Press, 2002).

[47] H. Haken, Laser theory (Springer-Verlag, 1984).

[48] H. Haken, Light: Laser light dynamics, Bd. 2 (NorthHolland Publishing Company, 1985).
[49] I. Protsenko, P. Domokos, V. Lefèvre-Seguin, J. Hare, J. M. Raimond, and L. Davidovich, Phys. Rev. A 59, 1667 (1999).

[50] C. Z. Ning, IEEE J. Sel. Top. Quantum Electron. 19 (2013).

[51] K. J. McNeil and D. F. Walls, J. Phys. A. 8, 104 (1975)

[52] B. Saleh and M. Teich, Fundamentals of Photonics (Wiley, 2007). 